\title{
Minimally invasive transxiphoid approach for management of pediatric cardiac tamponade - one center's experience
}

\author{
Ireneusz Haponiuk ${ }^{1,2}$, Ewelina Kwasniak ${ }^{1}$, Maciej Chojnicki ${ }^{1}$, Radoslaw Jaworski ${ }^{1}$, Mariusz Steffens ${ }^{1}$, \\ Aneta Sendrowska ${ }^{1}$, Katarzyna Gierat-Haponiuk ${ }^{3}$, Katarzyna Leszczyńska ${ }^{4}$, Konrad Paczkowski ${ }^{1}$, Jacek Zielinski ${ }^{5}$ \\ ${ }^{1}$ Department of Pediatric Cardiac Surgery, Mikolaj Kopernik Hospital, Gdansk, Poland \\ ${ }^{2}$ Chair of Physiotherapy, Gdansk Academy of Physical Education and Sport, Gdansk, Poland \\ ${ }^{3}$ Department of Rehabilitation, Medical University of Gdansk, Gdansk, Poland \\ ${ }^{4}$ Department of Obstetrics, Medical University of Gdansk, Gdansk, Poland \\ ${ }^{5}$ Department of Surgical Oncology, Medical University of Gdansk, Gdansk, Poland
}

Videosurgery Miniinv 2015; 10 (1): 107-114 DOI: $10.5114 /$ wiitm.2014.47690

\begin{abstract}
Introduction: Cardiac tamponade is excessive collection of fluid in the pericardial sac surrounding the heart that leads to restriction of cardiac function and causes critical cardiogenic shock and rapid circulatory depression. Despite the potential variety of different etiologies in the face of a dangerous decrease of cardiac output, the emergency life-saving procedure is surgical pericardial fluid evacuation.

Aim: To perform a retrospective analysis of clinical data and the results of minimally invasive transxiphoid pediatric cardiac tamponade evacuation procedures performed in a cardiac surgery center.

Material and methods: We performed a retrospective analysis of all consecutive patients referred for treatment in our department in a period of 6 years (15 patients) who underwent emergency pericardial drainage after an echocardiographically proven diagnosis. The procedure of choice was minimally invasive transxiphoid fluid evacuation with routine pericardial drainage. Retrospective operative data analysis was performed: clinical symptoms, pre-admission and initial emergency diagnostics and interventions, the morphology and total amount of drained pericardial fluid, length of stay, final results, and overall survival rate. We introduced an original pediatric tamponade index (PTI). The PTI was analyzed according to catecholamine support before the drainage and the length of hospital stay after the procedure.

Results: All patients survived the procedure. No early complications of the presented minimally invasive subxiphoid approach were noted. Mean PTI in patients with intensive catecholamine support before the operation was significantly higher than in patients without it.

Conclusions: Minimally invasive surgical transxiphoid interventions appear to be a safe and effective method to provide life-saving support with retrieval of the fluid for further laboratory investigations.
\end{abstract}

Key words: pediatric cardiac surgery, cardiac tamponade, viral infections, chest trauma.

\section{Introduction}

Cardiac tamponade is the excessive collection of fluid in the pericardial sac surrounding the heart that leads to restriction of cardiac function, and if massively grooving causes critical cardiogenic shock and rapid circulatory depression [1]. Although cardiac tamponade in children is a rare emergency, the

\section{Address for correspondence}

Dr. Radoslaw Jaworski, Department of Pediatric Cardiac Surgery, Mikolaj Kopernik Hospital, 1-6 Nowe Ogrody St, 80-803 Gdansk, Poland, phone: +48602 243 114, e-mail: radicis@go2.pl 
pathology can be found even in newborns, as well as being diagnosed prenatally. Pediatric tamponade could be related to previous pericardial interventions, or appear spontaneously, in patients without any history of cardiac problems. The intensity of pericardial fluid ingrowth and the dynamics of symptoms define two different forms: acute and chronic tamponade [1]. With regard to the time after the surgical procedure there is an early (up to 7 days after surgery) and late (over 7 days) tamponade, which appear up to 6 months after cardiac intervention [2]. Potential causes of non-surgical tamponade in children are heart failure (HF), infections, malignancies, collagen tissue diseases, chest trauma, hypothyroidism, uremia, and rheumatic fever (RF) [3, 4].

The pathophysiological consequences of pericardial fluid accumulation are initially the increase of intrapericardial pressure and the impairment of ventricular diastolic function. The increase of intrapericardial pressure causes compression on the atria with a restriction of atrial diastolic blood filling, and afterwards compression on the ventricles with a reduction of end-diastolic volume. The result is stroke volume reduction and impaired coronary perfusion, which cause the deterioration of cardiac output and activation of the sympathetic system with tachycardia. The increase of peripheral resistance and the afterload leads to reduction of end-diastolic volume, and additionally worsens cardiac output in a 'morbid circle' of cardiogenic shock. Clinical symptoms of cardiac tamponade in children and adults are known as Beck's triad: systemic hypotension, lowered heart sounds, and jugular venous overflow. There is evident systemic hypotension with high central venous pressure, dyspnea, tachycardia, impaired peripheral perfusion and paradox pulse with the reduction of pulse during intake of breath $[1,4]$.

Currently the diagnosis must be proved by transthoracic echocardiography before emergency intervention. Despite the potential variety of different etiologies in the face of a dangerous decrease of cardiac output, the emergency life-saving procedure is surgical, or alternatively interventional pericardial fluid evacuation when diagnosed. Limited tolerance for stroke volume reduction and life-threatening compression in children makes the emergency elimination of fluid even more urgent than in adult patients.

Following the literature reports as well as our own data analysis, there is an evident tendency to refer children with suspected pericardial tamponade for emergency treatment in pediatric cardiovascular centers. Although tamponade is not limited to patients with congenital heart defects we noticed some kind of 'good practice' to refer children with the diagnosis of pericardial fluid accumulation directly to cardiac surgeons. For that reason we need to follow commonly accepted and available standards that will be optimal and effective to help patients admitted to the institution in emergency settings.

\section{Aim}

The aim of the study was to perform a retrospective analysis of clinical data and the results of minimally invasive transxiphoid pediatric cardiac tamponade evacuation procedures performed in a pediatric cardiac surgery center.

\section{Material and methods}

We performed a retrospective analysis of all consecutive patients referred for the treatment in the Department of Pediatric Cardiac Surgery Mikotaj Kopernik Hospital in Gdansk, Poland, in the period from 1 January 2008 to 30 December 2013 (6 years). There were 15 cases of tamponade evacuation, constituting $1.1 \%$ of the total of 1372 pediatric cardiac procedures performed in the time frame.

The analyzed group consisted of 15 consecutive patients ( 5 males, 10 females) aged from 2 weeks to 18 years (average: 4.9 years) who underwent emergency pericardial drainage after an echocardiographically proven diagnosis of pericardial tamponade. Five patients had tamponade with shortness of breath and tachycardia, 4 complained of chest pain, and 5 had fever. Three children were immediately admitted from the Emergency Department directly to the Pediatric Intensive Care Unit because of a poor general condition and cardiogenic shock; they needed respiratory support and 2 of them - intensive catecholamine support. A 2-week-old newborn underwent pericardial puncture in the referring peripheral hospital; the attempts to evacuate the tamponade with needles resulted in cardiopulmonary resuscitation (CPR) and severe deterioration of general condition. Another critically ill baby girl received CPR in our Emergency Department just after admission. The diagnostics and the drainage were done while intensive resuscitation (closed chest heart massage) was continued up to $60 \mathrm{~min}$ after 
she entered the hospital. The initial clinical data of all referred patients are shown in Table I.

The procedure of choice at our institution was minimally invasive transxiphoid fluid evacuation with routine pericardial drainage left in place for a minimum of $24 \mathrm{~h}$ after the procedure. Meticulous retrospective operative data analysis was performed: clinical symptoms, pre-admission and initial emergency diagnostics (Photo 1) and interventions, and after the drainage: the morphology and total amount of drained pericardial fluid, length of stay, final results and overall survival rate.

In order to supplement the study we introduced an original pediatric tamponade index (PTI), a numeric relation between the total volume of drained fluid $(\mathrm{ml})$ during the time of pericardial drainage, and the initial body weight $(\mathrm{kg})$ of the patient. The PTI analysis was afterwards evaluated according to catecholamine support before the drainage, as well as the length of stay after the procedure.

Initially we assumed that miniinvasive surgical drainage would be our routine approach for emergency tamponade in pediatric patients of all ages. The arguments were an easy job for the pediatric cardiac surgeon, the safety of the drainage, and $24 \mathrm{~h}$ operating theatre availability. The decision was made in contrast to the risk of percutaneous pericardial puncture, and the complications observed especially in small babies. Surgical intervention was performed in the operating theatre under general anesthesia. The patients were prepared in a regular supine position with an elevating roller in the mid-thoracic area. The chest was opened via the subxiphoid minimally invasive median ministernotomy approach. The skin incision was no longer than 3-4 cm, with a separate stab wound for final chest tube insertion. After meticulous dissection of the retrosternal pericardial space the pericardial sac was opened and tension fluid was evacuated after initial puncture and aspiration, with dynamic outflow under pressure. In order to prevent acute cardiac dilatation, the decompression of pericardial tension was done gently, with the potential for the anesthesia team to initiate or elevate inotropic drug support. The fluid was assessed macroscopically (inflammatory, hemorrhagic, serofibrinous or serous exudate, lymphatic, suppurative, or bloodlike) and the volume was measured. The heart was dissected free from any fresh clots, fibrous layers, and adhesions, with regard to intraoperative find- ings. After the evacuation of fluid, direct-vision or digital pericardial inspection was performed with direct and palpable control of the heart and the inner surface of the pericardium. Any tumor, clots,

Table I. Characteristics of the patients who were referred with an initial diagnosis of pericardial tamponade

\begin{tabular}{|lc|}
\hline Parameters/anamnesis & Number/mean \\
\hline Gender & $\begin{array}{c}5 \text { males (33.3\%)/ } \\
10 \text { females (66.6\%) }\end{array}$ \\
\hline Age [years] & $\begin{array}{c}4.93 \\
\text { (range: } 2 \text { weeks }-18 \text { years) }\end{array}$ \\
\hline Body weight [kg] & 23.4 \\
(range: $1.1 \mathrm{~kg}-63 \mathrm{~kg})$
\end{tabular}

IPPV - intermittent positive-pressure ventilation, *cardiac surgery, percutaneous intervention, pericardiocentesis, drainage, ${ }^{* *}$ sepsis, neoplasm, endocrinology instability, renal or liver failure, ${ }^{* * *}$ cardiopulmonary resuscitation before or at admission, ${ }^{* * * *}$ adrenaline, noradrenaline, dopamine, dobutamine.

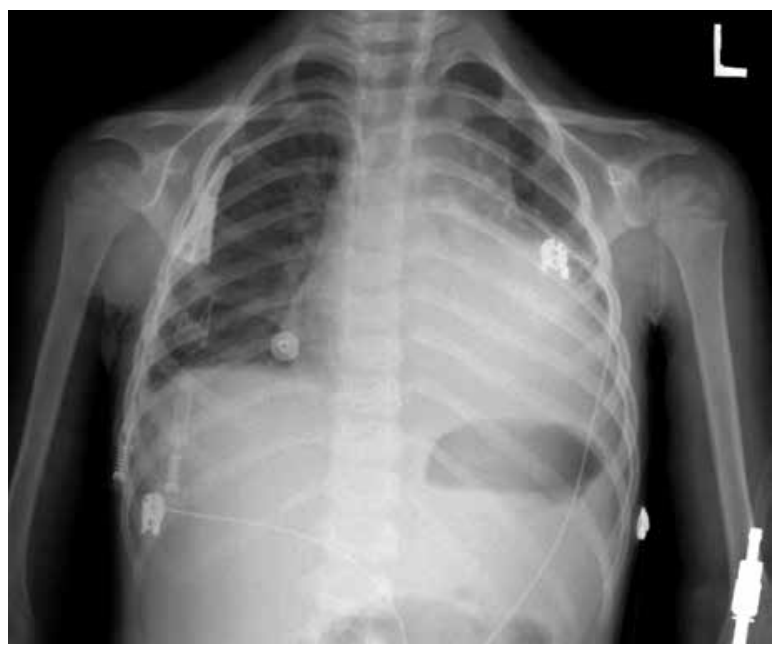

Photo 1. Preoperative chest X-ray with enormous heart silhouette -4.5 -year-old boy with postinfection pericardial tamponade 
or adhesions were thus identified. Patients with a longer history of a growing amount of pericardial fluids that finally caused a tamponade were additionally secured with a $2-3 \mathrm{~cm}$ wide fenestration to the right pleura in the angle between the diaphragm and right-sided mediastinal pericardial wall. The specimen from the fluid, any suspicious tissue, clots, and the pericardium were harvested for further laboratory and histopathology tests. The pericardial sac was flushed with warm saline and left open. The chest tube with active postoperative suction was left in the pericardial sac underneath the inferior wall of the heart (Photo 2), and the wound was closed in a routine manner.

All patients but two newborns were routinely extubated directly in the operative room, immediately after the procedure. Postoperative echocardiography, chest X-ray, and elective computed tomography in patients referred with malignant history were performed on the operative day. The chest tube was removed after $24 \mathrm{~h}$ routinely, unless there was persistent or continuous pericardial fluid evacuation. After the drainage, pharmacologic therapy with 3$5 \mathrm{mg} / \mathrm{kg} /$ day of oral acetylsalicylic acid was continued for 2-4 weeks in patients with exudative or inflammatory fluid, except for patients with blood-like tamponade.

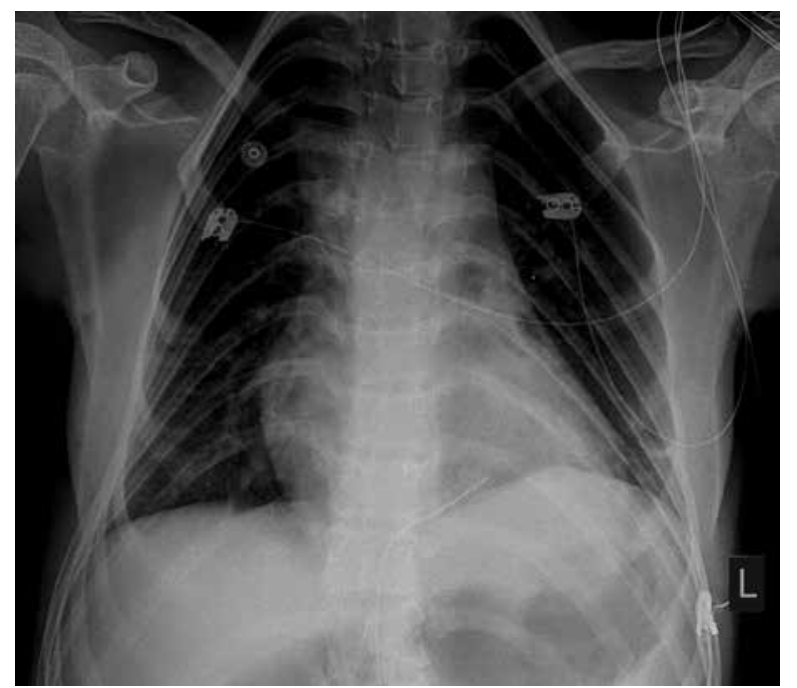

Photo 2. Postoperative chest X-ray with the drain under the base of the heart, inserted in diaphragmatic wall of the pericardial sac 13-year-old girl with tamponade in the course of pneumonia treatment

\section{Results}

All patients survived the procedure. There was one early death (early mortality 6.6\%) - a newborn with noncompaction of the left ventricle referred for tamponade evacuation with critical heart failure. No early complications of the presented subxiphoid approach were noted. There was no additional catecholamine support or reduction of initial drug support. The longest postoperative mechanical ventilation time was $18 \mathrm{~h}$. There was no long-term observation mortality or morbidity related to the procedure.

The time of total in-hospital stay after the drainage ranged from 1 to 19 days, average: 4.2 days. The shortest one-day-long hospitalization in our department was in fact continued in the referring pediatric hospital. The baby with fatal congenital cardiomyopathy has died 2 days after the drainage.

In every patient admitted with the diagnosis of cardiac tamponade there was a significant amount of pericardial fluid surrounding the heart, with compression on the right atrium and the right ventricle in the initial echo examination. The echocardiographic findings of critical tamponade with circulatory deterioration represented the "swinging heart" phenomenon. Although the fluid evacuated from the pericardium in 8 patients was blood-like (53.3\%), after further diagnostics a neoplastic process was diagnosed in 1 case (6.6\%). Three patients had inflammatory exudate, and 1 child had tuberculosis. The final diagnosis in the oldest teenage girl was lupus erythematosus, with the first manifestation of bloody pericardial tamponade. In the neonate with a history of prenatally diagnosed tamponade, who underwent emergency puncture just after birth in the referral neonatology department long before admission to the cardiac surgery center, finally the diagnosis of congenital parvoviral infection was established. Another 10-month-old boy with a congenital heart defect was admitted with cumulated chyle in the pericardial sac (chylopericardium). While the procedure of Gerbode-type ventricular septal defect (VSD) closure there was found an accompanying pericardial defect with a positional anomaly of the thoracic duct.

Five patients from the group (33.3\%) underwent previous cardiac surgery or cardiac intervention, with the timeframe from the initial procedure to 
pericardial drainage ranging from 4 to 56 days (average: 26.8 days), and 3 patients were subjected to pericardial puncture or drainage in referring hospitals (20\%) before admission. In 8 patients the fluid was blood-like, while in 1 patient chylopericardium was found. Fluid accumulation in the early period (7 days after the operation) was diagnosed in 1 child (6.6\%), while late tamponade appeared in 4 individuals (26.6\%).

The analysis of the pediatric tamponade index (PTI) was performed. The PTI analysis is shown in Table II. Mean PTI in patients with intensive catecholamine support before the operation was significantly higher than in patients without it: 42 (SD = 3.96; range: $39.1-44.7$ ) and 20.7 (SD = 11.6; range: $1.8-38.1)(p=0.027)$ respectively. No differences in PTI values were observed in children with postoperative hospitalization time longer than 2 days (mean PTI 23.9; SD 13.4 vs. 23.4; SD 13.7 in patients with maximal 2 days postoperative hospitalization period, $p=1$ ), as well as in patients with bloody-like vs. non-bloody like pericardial fluid (mean PTI 19.1;
SD 15.1 and 28.6; SD $=8.85$ respectively, $p=0.132$ ) and in patients with previous cardiac interventions vs. without invasive treatment (mean PTI 18.2; $\mathrm{SD}=16.5$ and $26.2 ; \mathrm{SD}=11$ respectively, $p=0.27$ ). Pearson's correlation coefficient for PTI and postoperative hospitalization time was 0.323 , but was not statistically significant $(p=0.24)$.

\section{Discussion}

In our experience the procedure of choice in pericardial tamponade is surgical pericardial drainage via a minimally invasive transxiphoid approach with direct vision control of the pericardial sac and the heart. Apart from the analysis of our institutional data we want to discuss our strategy with other methods of pediatric tamponade evacuation, with the main concern for the most popular emergency percutaneous pericardial puncture.

Acute cardiac tamponade is a life-threatening emergency. Nevertheless its clinical course can be a challenge for diagnostics and therapy, which need

Table II. Clinical data and their impact on PTI - consecutively, in order of appearance

\begin{tabular}{|c|c|c|c|c|c|c|}
\hline Patient no. & Age & Weight [kg] & Volume of fluid [ml] & CAT & LOS [days] & PTI \\
\hline 1 & 5.2 years & 16 & 200 & - & 1 & 12.5 \\
\hline 2 & 4.5 years & 17 & 345 & - & 2 & 20.3 \\
\hline 3 & 47 days & 4.1 & 123 & - & 2 & 30.3 \\
\hline 4 & 4 months & 4.2 & 160 & - & 2 & 38.1 \\
\hline 5 & 1.5 years & 10.6 & 215 & - & 11 & 20.3 \\
\hline 6 & 2 months & 63 & 115 & - & 0 & 1.8 \\
\hline 7 & 1.6 years & 13 & 100 & - & 3 & 7.7 \\
\hline 8 & 39 days & 3.5 & 130 & - & 0 & 37.1 \\
\hline 9 & 12.3 years & 55 & 1990 & - & 19 & 36.2 \\
\hline 10 & 4 months & 4 & 65 & - & 7 & 16.25 \\
\hline 11 & 14 days & 1.1 & 20 & - & 1 & 18.2 \\
\hline 12 & 15.6 years & 52 & 640 & - & 1 & 12.3 \\
\hline 13 & 2 months & 3.8 & 170 & + & 2 & 44.7 \\
\hline 14 & 18 years & 46 & 1800 & + & 11 & 39.1 \\
\hline 15 & 14 years & 57 & 1040 & - & 1 & 18.3 \\
\hline Mean (SD) & 4.93 years (6.6) & $23.4(23.6)$ & $474(635)$ & - & $4.2(5.5)$ & $23.5(13.1)$ \\
\hline
\end{tabular}

CAT - catecholamine support before the drainage, LOS - length of stay, hospitalization related to cardiac tamponade, PTI - pediatric tamponade index, SD - standard deviation. 
meticulous clinical examinations and vigilance to prevent oversight of potentially hazardous symptoms and terminal diseases. Acute tamponade, especially with bloody fluid, always requires oncological diagnostics of potential neoplastic growth $[5,6]$.

There is still a limited number of pediatric tamponade treatment reports in the literature. The groups reported number from 10 to 14 patients, while the adult papers analyze hundreds of cases in particular adult centers [4]. There are some reports of acute bloody pericardial tamponade in immunocompetent children due to viral infection [5] or general sepsis. Similar reports present children with rapid pericardial tamponade; most of them are patients with circulatory insufficiency due to heart failure, infections, mediastinal neoplasms and local compression or trauma of the heart [7-9].

The paper by Ozturk et al., dating from 2014, is based on the analysis of 14 pediatric patients treated with pericardiocentesis, mean age 7 years and mean body weight $26 \mathrm{~kg}$ [4]. Apart from the different population (older children), the authors were concentrated on the etiology of the pericardial fluid concentration, and their approach was pericardial puncture. Following the literature reports of fluoroscopy-controlled interventional fluid evacuation, although less invasive, it is difficult to perform even in referring centers because of the need for advanced technical equipment, with the great disadvantage of pediatric exposure to radiation. We did not consider this option despite the continuous availability of interventional resources and reported good results. We would keep our presented strategy for further babies referred with cardiac tamponades.

There are different strategies for cardiac tamponade reported in the literature $[3,10]$. The presented minimally invasive transxiphoid approach proved safe and effective tamponade evacuation in all referred children. We honestly choose surgical drainage, although this method is commonly reported as the second step, or a definitive procedure after initial pericardial puncture. Our arguments are that this approach enables pericardial inspection, dissection of pericardial adhesions, pericardial flush and macroscopic evaluation of the heart. The subxiphoid approach has the advantage of creation of a pericardial window to evacuate the fluid to the right pleura in case of any further accumulation [8]. Moreover, 3 babies who underwent transxiphoid drainage were previously subjected to needle puncture, which appeared only a temporary solution. It is usually reported that the pericardiocentesis approach can be complicated by rupture or puncture of the heart or coronary vessels, aeroembolism, pneumothorax, arrhythmias and puncture of the peritoneum or the organs in the upper abdomen [1].

From the technical point of view, we believe that in skilled teams this method is related to lower risk of complications [10]. Pericardial miniinvasive drainage is not difficult to perform in a cardiac surgery center. An additional advantage is mediastinal exploration with effective fluid evacuation in emergency, which makes transxiphoid ministernotomy the recommended procedure of choice in many centers. Three patients from the group were subjected to initial pericardial puncture, while in 2 cases the attempts to evacuate the fluids with needles caused the deterioration of the child.

In the presented group an emergency miniinvasive chest drainage was also necessary to save and stabilize the patients with accompanying comorbidity, who were previously subjected to continued antibiotic chemotherapy (infections, oncological therapy), as well as those who had no medical history. Both groups were referred for further diagnostics and follow-up analysis. Great interest was focused on the individuals with an unclear clinical picture observed while undergoing perioperative treatment. In patients who were drained in rapid emergency the diagnostics for thoracic tumor, general neoplasms, general viral infections, pneumonia, and any chest trauma were completed. According to the literature reports there is still a large group of patients who undergo an emergency pericardial tamponade evacuation without an initial or final diagnosis that could indicate the cause.

Good early results and minimal trauma related to miniinvasive techniques enabled early extubation and, simultaneously with planned anesthesia, weaning the patients off mechanical ventilation, with no complaints from the patients that could be related to the subxiphoid approach. That corresponds with available contemporary literature reports and emphasis evolving role of miniinvasive techniques in selected group of patients [11-15]. In patients with a previous history the procedure was the turning point for continuation of therapy, as well as for precise histopathological diagnosis and subsequent modification of the further strategy of care $[5,16,17]$. 
Our original parameter PTI enabled the assignment of presented patients to subgroups. We found that $\mathrm{PTI}>21$ was related to a more critical initial condition of referred patients (inotropic support), and might be correlated to longer length of stay after the drainage. This method was introduced especially for the purpose of this study and needs to be evaluated in further studies with more patients, but probably we will follow the PTI formula in further cases to prove its prognostic value and improve patient care.

With regard to the percentage of patients with a history of pericardial interventions, there is no doubt that previous cardiac treatment elevates the risk of cardiac tamponade. In the face of completed data from 1342 pediatric cardiac procedures in this institution, the meticulous controls with precise echocardiographic imaging are undoubtedly necessary to prevent late complications of the patients with a history of cardiac interventions.

Finally there comes the need to focus on the newborns and small infants who presented with pericardial tamponade in our report, who are the greatest age group treated for congenital heart defects in our institution. The presence of pericardial tamponade in neonates usually indicates a poor prognosis and is often related to heart failure, restrictive cardiomyopathy in fetal life, associated malformations, chromosomal abnormalities, fetal infection, intrauterine growth restriction, nonimmune hydrops, erythroblastosis, or central venous cannulation [18]. In prenatally diagnosed babies cardiac tamponade may lead to cardiogenic shock in the first hours after birth. According to our experience with unclear etiology of pericardial tamponade we suggest that in spite of the gestational history and pregnancy course, any neonate with unexplained fetal pericardial effusion should be tested for PV-B19 infection, even in the absence of known and proved fetal exposure. In our group 2 newborns (12.2\%) suffered from complicated pericardial puncture prior to forthcoming surgical pericardial drainage. Both procedures are extremely rarely performed in the newborn population, but according to the analysis above pericardial puncture appeared only as a temporary option to diminish the fluid compression in life-threatening circumstances. The emergency transxiphoid approach was effective in all newborns and infants referred with tamponade; thus we suggest that pericardial punctures in small babies is still questionable.

\section{Conclusions}

Pediatric cardiac tamponade in children could be the effect of many causes and is a life-threatening condition that needs an emergency treatment. Therefore pericardial drainage should be performed without any delay after an echocardiography confirmation. Despite usually unknown etiology of a critical tamponade in newborns and small infants, surgical transxiphoid interventions appear to be a safe and effective method to provide life-saving support with retrieval of the fluid for further laboratory investigations. The superiority of the transxiphoid pericardial approach consists in its safety and effectiveness in children of all ages with critical tamponade, while an additional advantage is pericardial inspection, which besides good final results could be crucial for further diagnostics and treatment.

\section{Conflict of interest}

The authors declare no conflict of interest.

\section{References}

1. Jaworska-Wilczyńska M, Kuśmierczyk M, Abramczuk M, Hryniewiecki T. Post-cardiac injury syndrome. Kardiochir Torakochir Pol 2013; 10: 20-6.

2. Yüksel V, Huseyin S, Canbaz S, et al. Emergency reoperation after open heart surgery: ten years of experience. Kardiochir Torakochir Pol 2013; 10: 195-8.

3. Kabukcu M, Demircioglu F, Yanik E, et al. Pericardial tamponade and large pericardial effusions: causal factors and efficacy of percutaneous catheter drainage in 50 patients. Tex Heart Inst J 2004; 31: 398-403.

4. Ozturk E, Cansaran IT, Saygi M, et al. Evaluation of non-surgical causes of cardiac tamponade in children at a cardiac surgery center. Pediatr Int 2014; 56: 13-8.

5. Haponiuk I, Chojnicki M, Jaworski R, et al. Bloody pericardial tamponade in a child treated for pneumonia mimicking a lung tumor and infiltration of the heart. Contemp Oncol 2013; 17: 225-7.

6. Perdeus P, Werner B, Kaliciński Z. Cardiac tamponade in a neonate with cardiac tumour. Kardiol Pol 2006; 64: 309-11.

7. Koranyi K, Yontz D, Rohrer Z, et al. Pericardial effusion complicating novel influenza A (H1N1) infection in an infant. Pediatr Infect Dis J 2010; 29: 782-3.

8. Tan BK, Pothiawala S, Ong ME. Emergency thoracotomy: a review of its role in severe chest trauma. Minerva Chir 2013; 68: 241-50.

9. Priola AM, Priola SM, Cardinale L, et al. The anterior mediastinum: diseases. Radiol Med 2006; 111: 312-42.

10. Becit N, Unlu Y, Ceviz M, et al. Subxiphoid pericardiostomy in the management of pericardial effusions: case series analysis of 368 patients. Heart 2005; 91: 785-90. 
11. Rawlinson E, Bagshaw O. Anesthesia for children with pericardial effusion: a case series. Paediatr Anaesth 2012; 22: 1124-31.

12. Haponiuk I, Chojnicki M, Jaworski R, et al. Gdansk Hybrid Heartlink Programme (GHHP) - we need to share the experience with miniinvasive hybrid procedures in borderline babies. Videosurgery Miniinv 2013; 8: 238-40.

13. Rachwalik M, Plonek T, Kustrzycki W, et al. The use of minimally invasive videoscopic technique in large vessel and cardiac surgery. Does the potentially increased difficulty bring benefits to the patient? Videosurgery Miniinv 2013; 8: 86-9.

14. Haponiuk I, Chojnicki M, Steffens M, et al. Miniinvasive interventional bridge to major surgical repair of critical aortic coarctation in a newborn with severe multiorgan failure. Videosurgery Miniinv 2013; 8: 244-8.

15. Emrecan B, Ozdemir AC. Surgical pitfalls of minimally invasive direct coronary artery bypass procedure from the viewpoint of a surgeon in the learning curve. Videosurgery Miniinv 2013; 8: 74-9.

16. Arabi MT, Malek EM, Fares MH, Itani MH. Cardiac tamponade as the first manifestation of systemic lupus erythematosus in children. BMJ Case Rep 2012; Sep 2012 doi:10.1136/bcr-2012006927.

17. Jaworski J, Haponiuk I, Irga N, et al. Critical cardiac tamponade in newborn, life-saving emergency interventions and possibility of parvovirus B19 congenital infection. Centr Eur J Med 2013; 8: 420-3.

18. dos Santos Modelli ME, Cavalcanti FB. Fatal cardiac tamponade associated with central venous catheter: a report of 2 cases diagnosed in autopsy. Am J Forensic Med Pathol 2014; 35: 26-8.

Received: 9.08.2014, accepted: 1.12.2014. 